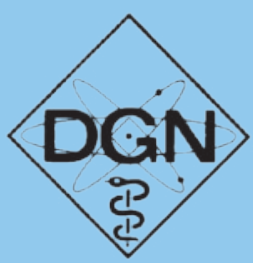

\title{
Neuer DGN-Vorstand nimmt seine Arbeit auf
}

Zum 1. Januar 2019 hat der neu gewählte Vorstand der DGN seine Arbeit aufgenommen.

Während die Professoren Krause, Luster, Freudenberg, Kotzerke, Schäfers und Essler dem DGN-Vorstand bereits angehörten, sind Frau Professor Patt und Pöpperl sowie Herr Professor Bartenstein und la Fougère in diesem Jahr neu hinzugekommen.

Die Zusammensetzung des neuen DGNVorstandes im Einzelnen:

\section{Prof. Dr. med. Bernd Joachim Krause}

Präsident

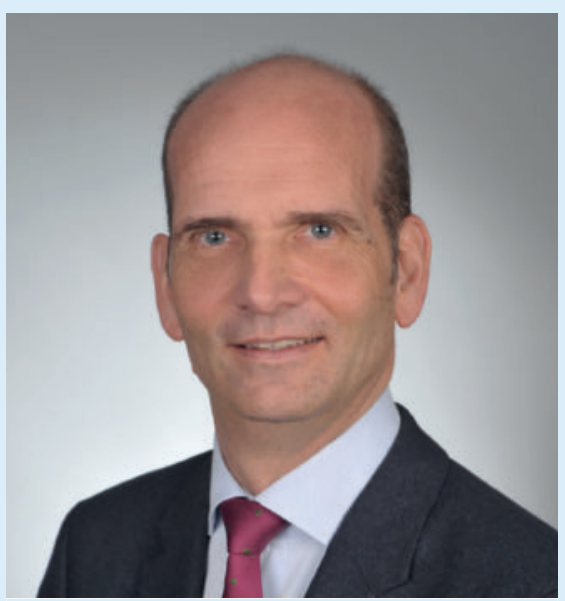

Prof. Dr. med. Bernd Joachim Krause (Quelle: privat)

Dem Vorstand der DGN gehört Herr Professor Krause seit dem Jahr 2013 an. Dabei nahm er von 2013 bis 2015 das Amt des stellvertretenden Vorsitzenden und Ausschusskoordinators wahr, bevor er mit dem Jahr 2016 als Präsident den Vorsitz der DGN übernahm.

Im Vorstand ist er zudem für den Bereich Leitlinien zuständig, den er bereits von 2011 bis 2015 als Vorsitzender des Ausschusses Leitlinien verantwortete.
Herr Professor Krause ist Direktor der Klinik und Poliklinik für Nuklearmedizin der Universitätsmedizin Rostock.

Er ist zudem als stellvertretender Delegierter der DGN bei der Arbeitsgemeinschaft der Wissenschaftlichen Medizinischen Fachgesellschaften (AWMF) tätig. Bis zum Februar 2018 war er Sprecher der Arbeitsgemeinschaft Bildgebung in der Onkologie (ABO) der Deutschen Krebsgesellschaft, seitdem hat er dort das Amt des Stellvertretenden Vorsitzenden inne.

Weiter war Herr Professor Krause von 20132016 Mitglied des Boards der European Association of Nuclear Medicine (EANM) und Committee Coordinator der EANM.

Prof. Dr. med. Markus Luster

Stellvertretender Präsident

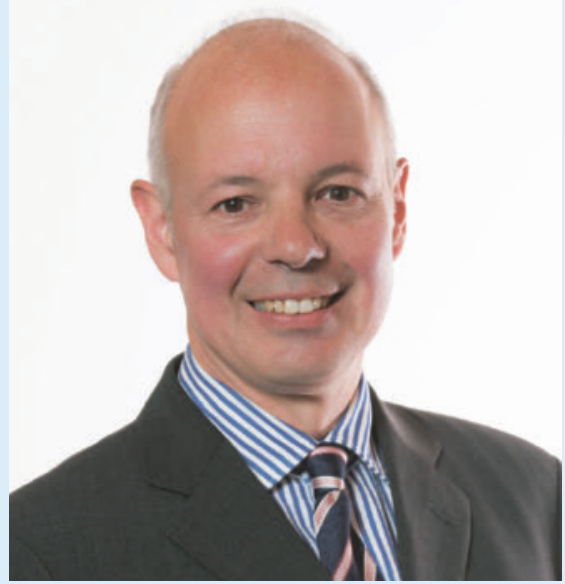

Prof. Dr. med. Markus Luster (Quelle: Janto Trappe (Hannover))

Herr Professor Luster ist Direktor der Klinik für Nuklearmedizin der Universitätsklinik Marburg. Er gehört dem Vorstand der DGN seit dem Jahr 2016 als Stellvertretender Präsident an. In diesem übernimmt er die Aufgabe des Ausschusskoordinators und ist da- rüber hinaus für die Bereiche Therapie und Schilddrüse zuständig.

Von 2012 bis zu ihrer Überführung in einen Ausschuss stand Professor Luster der AG Schilddrüse vor.

Im Moment ist er Vorsitzender des „Schilddrüsen-Komitees“ der Europäischen Gesellschaft für Nuklearmedizin (EANM) sowie Mitglied im wissenschaftlichen Beirat der Patientenselbsthilfegruppen „Ohne Schilddrüse leben e. V.“ (Deutschland) und „Butterfly Trust“ (Vereinigtes Königreich).

Prof. Dr. med. Peter Bartenstein

Schriftführer

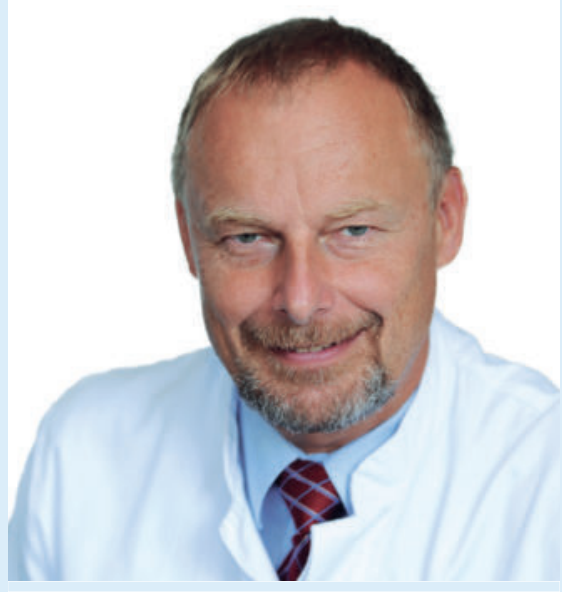

Prof. Dr. med. Peter Bartenstein (Quelle: privat)

Herr Professor Bartenstein ist Direktor der Klinik und Poliklinik für Nuklearmedizin der LMU München, Klinikum Großhadern.

Im Vorstand der DGN war er bereits von 2010 bis 2012 als Beisitzer tätig. In diesem war er für den Bereich Leistungserfassung verantwortlich. Darüber hinaus gehörte er bis 2015 dem DGN-Ausschuss PET an. Zudem ist er Mitglied der AG Neuronuklearmedizin. 
Seit 2008 ist Professor Bartenstein Mitglied des wissenschaftlichen Beirates der Bundesärztekammer sowie langjähriges Mitglied das Brain Imaging Council der Society of Nuclear Medicine. Zudem ist er Leiter der LMU Munich Medical Research School und Vorsitzender des Promotionsausschusses der Medizinischen Fakultät der LMU.

Prof. Dr. med. Dr. rer. medic. Lutz Stefan Freudenberg

Kassenführer

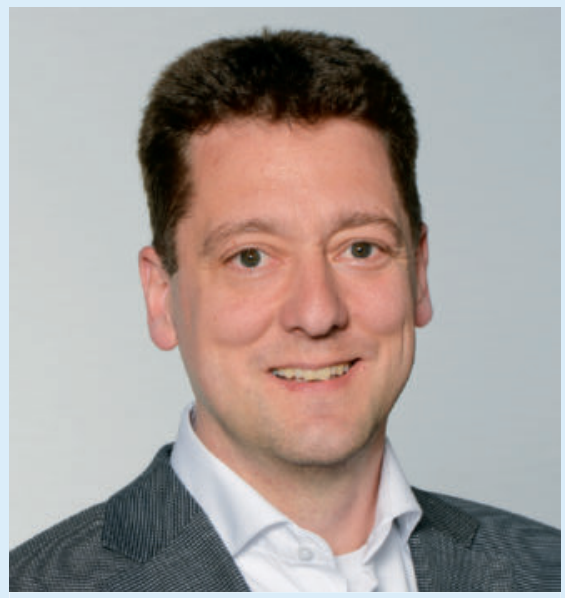

Prof. Dr. med. Dr. rer. medic. Lutz Stefan Freudenberg (Quelle: Frank Pusch (Bremen))

Herr Professor Freudenberg ist niedergelassener Facharzt für Nuklearmedizin im Zentrum für Radiologie und Nuklearmedizin Rheinland. Er hat zudem einen Lehrauftrag an der Universität Duisburg-Essen inne.

Seit 2010 ist Herr Professor Freudenberg Mitglied des Vorstands der DGN, wo er seitdem als Kassenführer tätig ist. Im Vorstand ist er auch für den Bereich Fort- und Weiterbildung zuständig.

Herr Professor Freudenberg war zudem von 2010 bis 2016 Delegierter der DGN zur Nuclear Medicine of the European Union of Medical Specialists (UEMS) und des European Board of Nuclear Medicine (EBNM).
Prof. Dr. med. Christian la Fougère Beisitzer

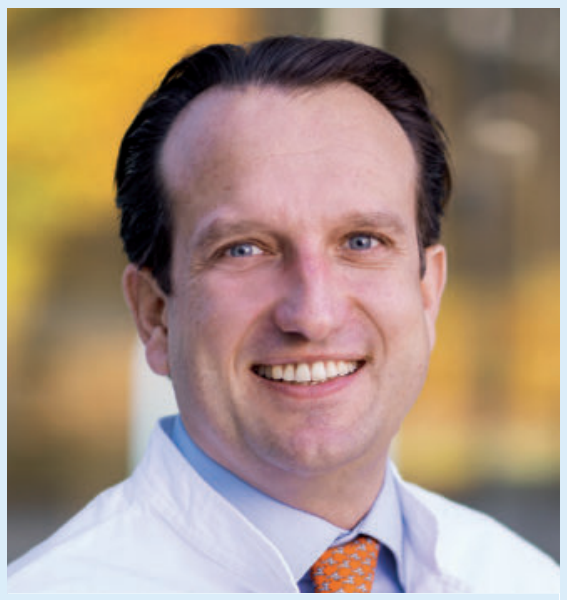

Prof. Dr. med. Christian la Fougère (Quelle: Universitätsklinikum Tübingen)

Herr Professor la Fougère ist Leiter der Abteilung Nuklearmedizin und Klinische Molekulare Bildgebung am Universitätsklinikum Tübingen.

In der DGN ist er seit 2016 Vorsitzender des Ausschusses Multicenter-Studien, zudem ist er Mitglied der AG Neuronuklearmedizin. Darüber hinaus ist er Mitglied der Sektion Schilddrüse der DGE.

Bei der NuklearMedizin 2018 in Bremen war er der Vorsitzende des wissenschaftlichen Komitees. Im Jahr 2015 war er zudem Kongresspräsident der SWDGN Jahrestagung in Tübingen.

Prof. Dr. rer. nat. Marianne Patt Beisitzerin

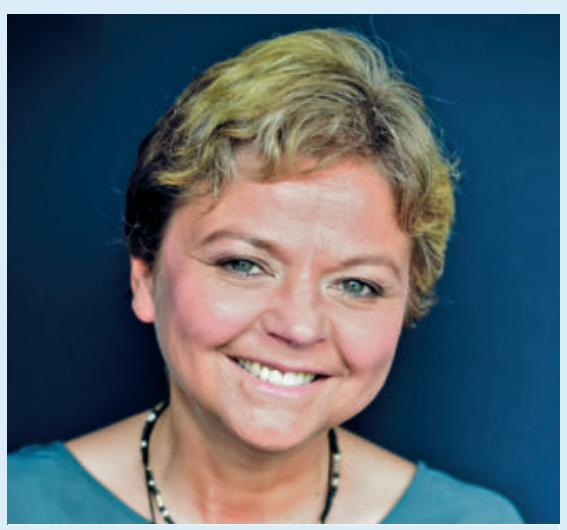

Prof. Dr. rer. nat. Marianne Patt (Quelle: privat)
Frau Professor Patt ist Leitende Radiochemikerin in der Arbeitsgruppe Radiochemie/ Zyklotron der Klinik und Poliklinik für Nuklearmedizin des Universitätsklinikums Leipzig.

Seit 2011 gehört sie dem DGN-Ausschuss Radiopharmaka an, dessen Vorsitz sie 2016 übernahm.

Darüber hinaus ist sie Mitglied in der $\mathrm{Ar}$ beitsgruppe Radiopharmaka beim Bundesamt für Arzneimittel und Medizinprodukte sowie Mitglied im Komitee der Arbeitsgemeinschaft Radiochemie/Radiopharmazie der DGN. Sie ist zudem seit 2015 Mitglied des „Radiopharmacy Committee“ der Europäischen Gesellschaft für Nuklearmedizin (EANM) und seit 2018 Vorsitzende dieses Gremiums.

Prof. Dr. med. Gabriele Pöpperl Beisitzerin

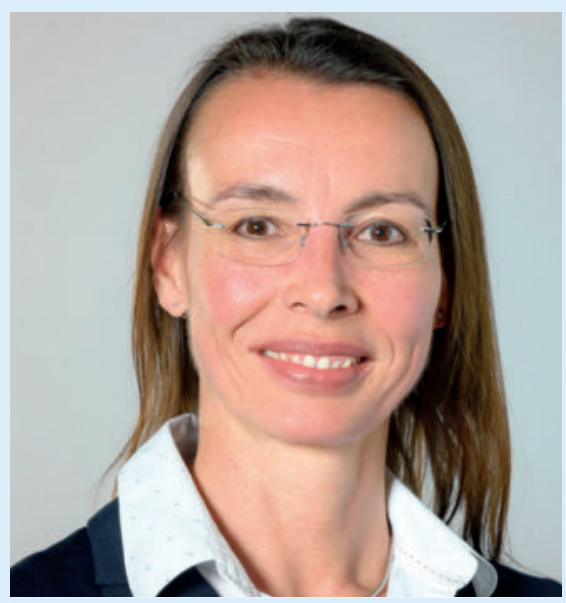

Prof. Dr. med. Gabriele Pöpperl (Quelle: Frank Pusch, Bremen)

Frau Professor Pöpperl ist Fachärztin für Nuklearmedizin und seit dem Jahr 2009 ärztliche Direktorin der Klinik für Nuklearmedizin des Klinikums Stuttgart. Als außerplanmäßige Professorin hat sie zudem einen Lehrauftrag an der Ludwig-MaximiliansUniversität München.

Frau Professor Pöpperl ist Mitglied des Ausschusses Therapie der DGN, des Vorstandes des Deutschen NET-Registers und berät außerdem die bundesweite Selbsthilfegruppe Netzwerk NET e. V. als Mitglied im wissenschaftlichen Beirat. Als Mitglied der SWDGN war sie Kongresspräsidentin der Jahrestagung der SWDGN 2018 in Stuttgart. 


\section{Prof. Dr. med. Jörg Kotzerke}

Altpräsident

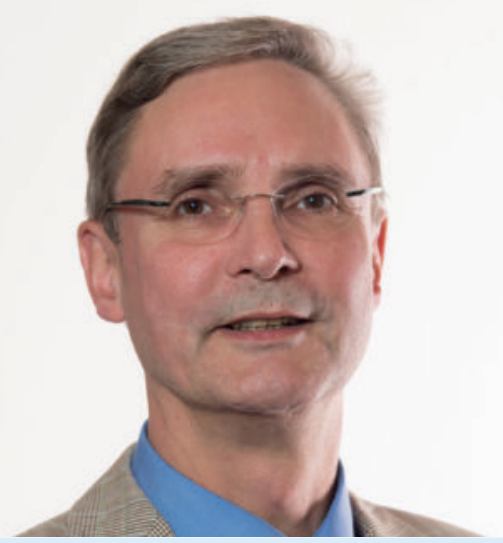

Prof. Dr. med. Jörg Kotzerke (Quelle: Janto Trappe (Hannover))

Herr Professor Kotzerke ist Direktor der Klinik und Poliklinik für Nuklearmedizin des Universitätsklinikums Carl Gustav Carus in Dresden. Dem Vorstand der DGN gehört er seit 2007 an. Zunächst war er als Schriftführer tätig, von 2010 bis 2015 war er Präsident der DGN. Bis 2009 hatte er für sechs Jahre auch den Vorsitz des Ausschusses PET inne.

Herr Professor Kotzerke ist zudem Schriftleiter der Zeitschrift Nuklearmedizin.
Prof. Dr. med. Michael Schäfers

Kongresspräsident 2019

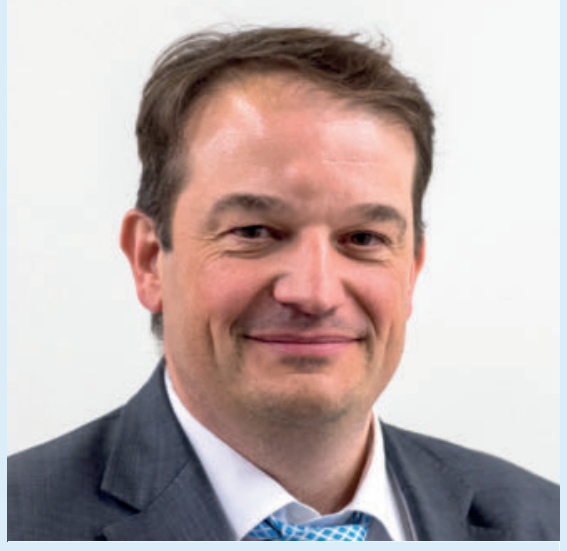

Prof. Dr. med. Michael Schäfers (Quelle: Christian Schneider-Bröcker (Leipzig))

Herr Professor Schäfers ist Direktor der Klinik für Nuklearmedizin am Universitätsklinikum Münster sowie Leiter des European Institute for Molecular Imaging (EIMI) an der Westfälischen Wilhelms-Universität Münster.

Er ist Mitglied der AG Kardiovaskuläre Nuklearmedizin und der AG 20 der Deutschen Gesellschaft für Kardiologie. Im Jahr 2019 fungiert Herr Professor Schäfers als Kongresspräsident der Jahrestagung der DGN in Bremen.
Prof. Dr. med. Markus Essler

Kongresspräsident 2020

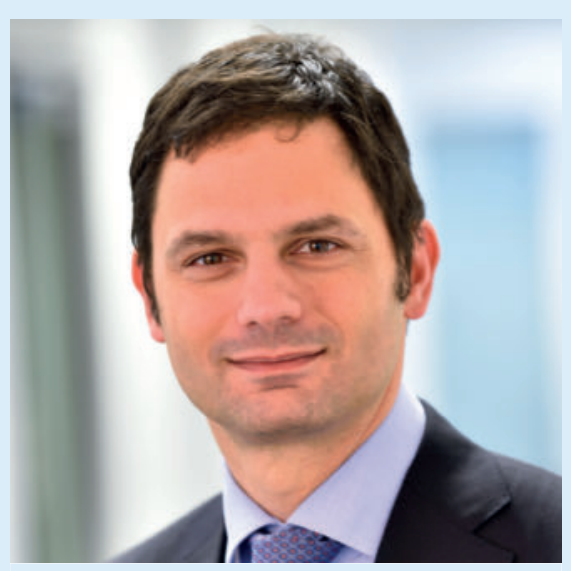

Prof. Dr. med. Markus Essler (Quelle: Universitätsklinikum Bonn (UKB))

Herr Professor Essler ist Direktor der Klinik und Poliklinik für Nuklearmedizin des Universitätsklinikums Bonn.

In den vergangenen Jahren hat sich Herr Professor Essler besonders für das „Reimbursement“ von innovativen Nuklearmedizinischen Therapieverfahren wie der SIRT und der ${ }^{177}$ Lu-PSMA-Therapie an den Universitätskliniken in Nordrhein-Westfalen engagiert und tritt auf nationaler und internationaler Ebene für die Durchführung von prospektiven Studien im Bereich der Nuklearmedizinischen Therapie ein. 